ein paarmal in den Personalakten stehen hat, er neige zum Widerspruch und so, dann können sie damit rechnen, daß im Moment bei dieser Großwetterlage dieser dann nicht in den Justizdienst enngestellt wird, weil sie sagen, aha, hier haben wir einen, der macht Stunk nachher. $D a$ jetzt die Bewerber quasi Schlange stehen, können sie sich die Leute sehr stark aussuchen. Da machen die natürlich von dieser Sache Gebrauch und das ist eben ganz gefährlich. Hier zeigt sich eine Existenzgefährdung, die den Leuten droht. wenn ste abgewiesen werden. Sie können höchsters noch in der Anwaltschaft unterkommen usw. Das ist eine Existenzangst bei den Assessoren, die Familie haben oder ähnliches. Wenn die rausfliegen bei der Lage auf dem Arbeitsmarkt im juristischen Sektor. der sichtbar schlecht ist hier in Frankfurt, bei einer Uberbesetzung von Anwälten usw. ist das eine Existenzbedrohung. Diese Dinge spielen auch eine große Rolle, die Existenzangst, die wird auch bewußt eingeführt, um all diese Dinge zu ersticken.

Interview und Aufzeichnung: Projektgruppe Mietrecht, Staatliche Wohnungspolitile und Mieterselbsthilfeorganisationen an der FU Berlin.

\title{
Die Praktiken des BND, oder: Was man aus dem Guillaume-Untersuchungsausschuß lernen kann.
}

Eigentlich muß man G. Guillaume dankbar sein, hat doch sein Fall wenigstens ein wenig den Schleier der Geheimniskrämerei gelüftet, der traditionellerweise die Praktiken der bundesdeutschen Geheimdienste umgibt. Angeregt durch das öffentliche Interesse an dieser "größten Spionageaffäre in der Geschichte der Bundesrepublik ${ }^{1}$ förderte sogar der zu ihrer Aufklärung eingesetzte parlamentarische Untersuchungsausschuß Interessantes zutage. Dies ist umso erstaunlicher, als in Bonn jedermann weiß, daß parlamentarische Untersuchungsausschüsse äußerst untaugliche Aufklärungsmittel sind. Bundeskanzler Schmidt äußerte sogar, die Einsetzung eines Untersuchungsausschusses in dieser Affäre gefährde "das Ansehen des Parlaments schlechthin, einschließlich der CDU/CSU. Untersuchungsausschüsse hätten bisher noch nie dazu beigetragen, die Achtung vor dem Bundestag zu verstärken. ${ }^{2}$ Die Richtigkeit dieses Urteils stellte sich schnell heraus, denn sorwohl CDU/CSU als auch ihr nahestehende Zeitungen versuchten, die Gelegenheit vor allem zu dem Versuch zu nutzen, die SPD und sogar Verfassungsschutzpräsident Nollau als vaterlandslose Gesellen zu "enttarnen«:

- "Fraktionschef Herbert Wehner, so deutet die Opposition an, habe dem Kommunismus nie abgeschworen und SED-Chef Honecker von der bevorstehenden Enttarnung Guillaumes am 30. März 1973 unterrichtet.

- Günther Nollau, von Wehner geförderter Chef des Kölner Bundesamtes für Verfassungsschutz (BfV), so läßt die Reaktion in der Zeitschrift "Capital“" andeuten, sei ein Agent des Ostens.

- Marie, die tschechische Ehefrau des ehemaligen Kanzleramtsministers Horst Ehmke, so wird kolportiert, sei eine Ostagentin und

\footnotetext{
1 Der Spiegel (DS) $18 / 74$, S. 19.

2 Frankfurter Rundschau (FR) 12.6. 1974 .
} 
- Entspannungsminister Egon Bahr, so wird suggeriert, habe nachrichtendienstliche Kontakte zur Sowjetunion unterhalten. Alles nach dem Motto: auch wenn es nicht wahr ist, es bleibt immer etwas hängen. " ${ }^{3}$

Diese "Erkenntnıs" wurde noch um eine Vielzahl weiterer Spekulationen, insbesondere durch „Quick", Springer-Presse und ZDF-Löwentha] angereithert. Sie gewähren einen interessanten Einblick in die Geistesverfassung und die Denunziationsmethoden der politischen Reaktion in der BRD. Allerdings konnte der aufmerksame Zeitungsleser im Zusammenhang mit dem GuillaumeUntersuchungsausschuß auch allerlei über die bundesdeutschen Geheimdienste lernen, das sich lohnt festzuhalten:

Der Bundesnachrichtendienst (BND), eigentlich nur für Spionage im Ausland zuständig, hat zumindest bis zum Regierungsantritt der SPD/FDP-Koalition auftragstwidrig mindestens $4+$ namentlich bekannte bundesdeutsche Politiker bespıtzelt und über sie Akten angelegt. Der "Stern" berichtet von einer insgesamt "600 Personen umiassenden Sonderkartei. «t Diese Dossiers rurden unkontrolliert fotokopiert und auch an Dritte weitergegeben.

Zur rechtichen Würdigung der BND-Praktiken ist es zunächst notwendig, darauf hinzuweisen, daß es eine gesetzliche Grundlage für den BND bis auf den heutigen Tag nicht gibt. Vielmehr wurde der BND »durch Kabinettsbeschluß vom II. Juli I95s am 1. April 1956 in Dienst gestellt... Grundlage dieses Beschlusses war die der Bundesregierung ipso jure zustehende Organisationsgewalt. ${ }^{5}$ Die nur in vertraulichen Kabinetrsbeschlüssen festgelegten Aufgaben des BND umriß BND-Präsident Wessel folgendermaßen: "Der BND sammelt und bearbeitet im Interesse der Sicherheit unseres Landes und im Auftrag der Bundesregierung im Ausland Nachrichten, die aus anderen Informationsquellen nicht zur Verfügung stehen und deshalb als Beurteilungselemente für die Entscheidungen der Regierung unentbehrlich sind. * Zum Bereich Beschaffung heißt es in den BND-Statuten: "Beschaffung von Informationen militärischen, wirtschaftlichen und rüstungstechnischen, sowie politischen Inhaits mit nachrichtendienstlichen Mitteln aus dem Ausland. " 6

Entgegen dieser klaren Beschränkung des BND auf Auslandsaufgaben bestätigten vor dem Untersuchungsausschuß der ehemalige Kanzleramtschef und BND-Dienstherr Horst Ehmke und BND-Präsident Gerhard Wessel BNDSpitzeldienste im Inland. Die entsprechenden Dossiers umfaßten nicht nur die politischen Aktivitäten der Bespitzelten, sondern ebenso ihre ${ }_{E} \beta-$, Trink- und Bettgewohnheiten. "Ehmke laut Spiegel: „Der frühere BND-Präsident "Gehlen * brauchte das, um sich allen Versuchen, den Dienst unter Kontrolle zu bringen, entziehen zu können. Woher wußte wohl Adenauer, daß sein FDP-Vize Blücher mal was mit'ner. Mulattin gehabt haben soll, wenn nicht vom BND. «? Nicht nur Einzelpersonen wurden überwacht, sondern auch Organisationen. Noch im März 1971, also zwei Jahre nach der Regierungsübernahme von SPD und FDP, flog eine BND-Außenstelle auf, die Material über die SPD sammelte. SPD-Interna - unter anderem Aufzeichnungen von vertraulichen Vorstandssitzungen ${ }^{8}$ - hatte der einstige Leiter des früher in der DDR nachrichten-

3 Konkret $2 ; 7+$, S. 6 .

+ Sern +1174 . S. 160 .

5 Thomas Walde: ND-Report. Die Rolle der geheimen Nachnchtendienste im Regrerungssystem der BRD, München 197 t. S. 79.

- Ebenda, S. sir f.

7 DS +2174 , S. 25 tf., die Namen von bespitzeleen Policikern veröffentlichte die FR an ihrer Ausgabe ium 10. 10. 1974 .

FR vom $23.10 .7+$ 
dienstlich arbettenden Ostbüros der SPD für den BND zusammengetragen.9 $\mathrm{Da}$ diese BND-Dossiers auch an Dritte, zum Beispiel an Parteien oder Journalisten, rveitergegeben wurden, hicht BND-Präsident Wessel für denkbar. ${ }^{10}$ SPDBundesgeschäftsführer Holger Börner hatte Anhaltspunkte dafür, daß "Ergebnisse von solchen Ausspähungen auch als innenpolitusche Knüppel« verwandt wurden. " Ehmke sagte aus, daß zum Beıspiel "das über die SPD vom BND sohne Auftrag der Auslandsaufklärung zusammengetragene Material interessierten politischen Kreisen zugänglich gemacht wurde. "12 Der frühere Chef der BND-Abteilung Beschaffung, General a. D. Langkau, bekannte, daß er Material über Persönlichkeiten des öffentlichen Lebens aus anfallenden Berichten zusammengefalßt und als sogenannte Führungsnotizen direkt an den damaligen BND-Präsidenten Gehlen weitergegeben habe, worauf die Originalunterlagen vernichtet worden scien, so daß\} eine spätere Kontrolle der nNotizen«, die Gehlen wahrscheinlich in seine Sonderkartei genommen habe, nicht mehr möglich gervesen sei. ${ }^{13}$

Neben Politikern, führenden Oppositionellen, wie etwa Wolfgang Abendroth oder Viktor Argartz ${ }^{14}$ und der SPD bespitzelte der BND auch noch ausgiebig Verlage, Journalisten und Verleger; so den "Stern" und den "Spiegel", den Springer- und Bauer-Verlag, Augstein und Nannen, Sebastian Haffner und o8/is - Autor Hans Hellmut Kirst, ${ }^{15}$ Margarethe Buber-Neumann und Gert Bucerius. ${ }^{16}$ Ein Star-Inlandsagent des BND in dieser Sache, Günther Heysing, beschaffte übrigens noch bis 1973 einschlägıges Materıal für den BND. ${ }^{17}$ Er beschränkte sich nicltt auf Materialbeschaffung, sondern zettelte durch heimliche Denunziationen und Tips an reaktionäre Organısatıonen aktiv Kampagnen etwa gegen Kirst oder Haffner an. Auch den BND animierte er "zu Praktiken psychologischer Kriegstührung. In Dokumentationen und durch stete Wiederholung von Vorwürfen, die darin gipfeln sollten, Haffner stehe im Sold der SED, müsse der Kommentator so unglaubwürdig gemacht werden, dal' selbst Herausgeber Bucerius das Vertrauen in seinen Mann verliere. Heysing an den BND: ,Eine Einschränkung der Wirksamkeit von $H$. kann nur errescht werden, wenn ihm die publizıstische und wirtschaftliche Basis beim Stern zerstört wird. « 18

Waren mit BND-Unterstützung innenpolitische Kämpfe zu führen, so richteten sich diese stets gegen die politische Linke, wie das oben genannte Beispiej bereits zeigt. Hatte R. Gehlen die Mitglieder seines Dienstes vorwiegend aus SD-, Gestapo- und Nachrichtenoffizieren der NS-Ära rekrutiert, so wurde die reaktionäre Gesinnung dadurch konstant gehalten, daß neue Mitarbeiter vorwiegend auf Empfehlung von Bekannten und Verwandten angeworben wurden. "Folge: In dem ohnehin schon konservatuven Nachrichtendienst bildeten sich immer neue rechtslastige Zirkel, "19 was auch eine Zusammenarbeit mit entsprechenden gesellschaftlichen Kräften rahelegt.

So überrascht es nicht, daß dic von dem BND-Agenten Bährvald - heute

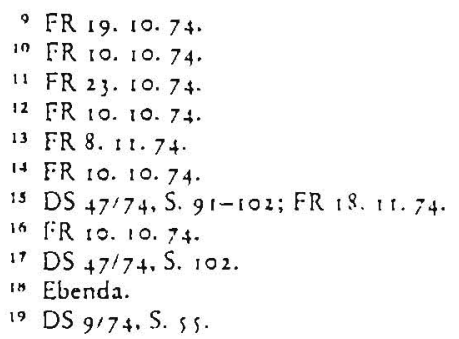


"Berater" der CDU/CSU - ausgespähten SPD-Interna der CSU-Landesleıtung in München zugespielt wurden 20 und daß $\mathrm{H}$. Ehmke nicht vom BND, sondern zuerst von F. J. Strauß erfuhr, daß bei einer BND-Außenstelle die Akte über SPD-Interna sichergestellt werden konnte. ${ }^{21} \mathrm{Zu}$ diesem Zusammenspiel zwischen BND-Mitgliedern und konservativen Kräften gehört weiter, daß

- "die CDU/CSU durch Indiskretionen aus ejnigen Bundesdienststellen in der Aftäre Guillaume über erheblich mehr Informationen als die Regierung verfügt , $^{22}$

- von Vertretern der CDU/CSU präzise Fragen zu Ermittlungskomplexen im Falle Guillaume gestellt wurden, die zu diesem Zeitpunkt von den Behörden noch geheimgehaiten wurden, ${ }^{23}$ bzw. bis dahın noch nıcht einmal dem Kanzleramt bekannt waren, ${ }^{24}$

- die CDU/CSU - nahe Presse Andeutungen über Ermittlungsergebnisse veröffentichte, bevor im Vertrauensmänner-Gremium die Abgeordneten darüber unterrichtet worden waren, ${ }^{25}$

- dem konservativen ZDF-Moderator Löwenthal BND-Geheimberichte zugespielt wurden, ${ }^{26}$

- BND-Mitarbeiter von Akten, die Ehmke zu vernichten befohlen hatte, Fotokopien anfertigten und an konservative Massenmedien weitergaben, ${ }^{27}$

- Erkenntnisse aus einer geheimen Verschlußsache der Führungsorientierung des BND bei der Fragestunde des Bundestages durch den CSU-Abgeordneten L. Niegel veröffentlicht wurden, ${ }^{28}$

- die "CSU-Seilschafu (Pullach-Jargon) ... noch heute die Personalpolitik des BND beeintlußt - trotz sozialliberaler Reformversuche "zy und es schaffte, die Antikommunistin und Anti-Brandt-Kämpferin S. Sievers als BND-Mitarbeiterin anzustellen ${ }^{30}$.

Ehmke: "Was den BND in den Ruf gebracht hat, mit der CSU verfilzt zu sein, ist das Zusammenspiel in vielen Kampagnen... Da liegt dann die Gefahr nahe, daß sich Teile dieses Dienstes verselbständigen und Mitarbeiter aut fremde Rechnung arbetten."31 Außer ehemaligen BND-Agenten sind übrigens auch solche des Verfassungsschutzes (VS) für die CDU/CSU tätig. ${ }^{32}$ Nur am Rande sei erwähnt, dal auch diese Praktiken auftragswidrig sind, denn es gehört naturgemäß nicht zu den Aufgaben des BND - zudem gegen den Willen der Bundesregierung - konservative Kräfte mit geheimem Material zu versorgen.

Der BND hat sich zur Beenflussung der öffentichen Meinung, zur eigenen Absicherung und zur Inlandsauthlärung Journalisten und andere "politisch einAußreiche Persönlichkeiten durch Zahlung von Gefälligkeitshonoraren und Ubernahme der Kosten für teure Vergnügungsreisen* verpflichtet. ${ }^{33}$ Dem

$20 \mathrm{FR}: 7,10.74$

$21 \mathrm{FR} 17.10 .74$.

22 FR 8. . . 74

2s [.R 6. $5 \cdot 7+$.

$\therefore$ DS $19 / 7+$, S. 21

is Ebenda.

so ER 15.6.74.

27 Ebenda.

28 FR 21.6 .74

24 DS $2117+$, S. 68

30 Ebenda. S. 72.

31 Extra-Dienst 29. 10.74, S. 10.

32 FR 12.9 .74$.

33 Seern $+117+$, S. is9. 
Stern-Bericht zufolge wagten es viele Politiker nicht, gegen den BND waufzutreten, weil er persönliches aus ihrer Privatsphäre in seinen Dossiers versammelt hatte. Und außerdem hatte er einflußreiche Leute durch finanzielle Zurvendungen so an sich gebunden, daß sie sich bei Bundeskanzler Adenauer, dessen Staatssekretär Globke und Knieber für ihn einsetzten. « Laut Ehmke, der von "übelster Korrumpierung von Teilen der Presse " durch den BND sprach, hat es seinerzeit erhebliche Zahlungen an Journalisten gegeben, die für den Dienst Inlandsaufklärung betrieben hätten. Er schilderte, daß Journalisten, die nebenher für den BND arbeiteten, gegen Honorar Berichte an BND-Außenstellen gaben und wenige Tage später unter Berufung auf diese Berichte Meldungen für ihre Blätter schrieben. " 34 Für diese Spitzeleien seien monatlich zwischen i $500,-$ und is $000,-D M$ - natülich steuerfrei - gezahlt worden. ${ }^{35}$ Der Redaktionsdirektor der Illustrierten "Quick " behauptete, daß "gut zehn Dutzend der führenden Journalisten in der Bundesrepublik Sonderverbindungen mit dem BND haben ", was eine "ehrenhafte * Sache sei, ${ }^{36}$ der Extra-Dienst weiß von 60 "BND-Mitarbeitern aus Journalistenkreisen " zu berichten. ${ }^{37}$ Laut Frankfurter Rundschau soll die "Führungsstelle für journalistische Mitarbeiter des BND... ihren Sitz seit Jahren in Neu-Isenburg südlich von Frankfurt haben. " ${ }^{38} \mathrm{Da}$ a sich Journalisten vom BND bestechen lassen, nannte BND-Chef Wessel eine "staatserhaltende Pflicht" ${ }^{39}$, General-Bundeanwalt a. D. Martin befand: "Was ist schon dabei? «10 Dagegen erhob der deutsche Presserat gegen diese Praktiken schärfsten Protest und drängte außerdem auf eine Änderung der wbisherigen Praxis(!), BND-Residenten im Ausland als Journalisten zu deklarieren. ${ }^{4 t}$

Der BND beteiligte sich am internationalen Waffenhandel und sorgte mit hoher Wahrscheinlichkeit mit dafür, daß unter Verstoß gegen das Außenwirtschaftsgesetz Kriegswaffen ohne die notwendige Genehmigung durch die zuständigen Bundesbehörden in Spannungsgebiete exportiert wurden. Uber die frühere Frankfurter Firma Radio A.I.R., nach dem Bericht des Fernsehmagazins "Monitor* eine Außenstelle des BND, und die Bonner Firma Merex AG scheint der BND mitgeholfen zu haben, illegale Rüstungsgeschäfte in Millionenhöhe zu tätigen und unter anderem über 100 Flugzeuge, sorvie Geschützc, Raketen, Maschinengewehre, Bordkanonen etc. an Saudi-Arabien, Indien und Pakistan zu liefern. ${ }^{+2}$ Das Kriegsmaterial stammte zum überwiegenden Teil aus überschüssigen Beständen der Bundeswehr und wurde aufgrund gefälschter Exportpapiere zur Ausfuhr freigegeben. Dem Spiegel zufolge soll nicht nur der BND, sondern auch der Staatssekretär des damaligen Bundeskanzlers Erhard, Ludger Westrick, über den Waffenhandel informiert gewesen sein. Auskünfte über diesen Waffenhandel wollten BND-Vertreter vor dem Untersuchungsausschuß nicht abgeben. Einzelheiten könnten nur in nichtöffentlicher Sitzung erörtert werden. Horst Ehmke allerdings antwortete auf die Frage $*$ Bestätigen Sie also, daß der BND Waffenhandel betrieben hat?": "Das ist vom Präsidenten (des BND) vor dem (Guillaume-) Ausschuß schon gesagt worden. “43 Selbst nach

34 FR 10. 10. 74 .

35 DS $44 / 74$.

36 FR 24.10 .74 .

37 Extra-Dienst vom 29. 10.74. S. 9.

$38 \mathrm{FR} 23.10 .74$.

$39 \mathrm{FR}: 5.10 .74 ; 10.12 .74$.

4) $\mathrm{DS}+5 / 74$, S. 8 .

$4 \mathrm{FR}+12.74$.

12 DS $47^{1 / 74}, 5.69-70$. FR v. 23.10 .74 , FR 12. 11. 74.

23 Extra-Dienst 29, 10. 74, S. 11 . 
Ansicht des früheren Kanzleramtschefs Carstens ist der Waffenhandel "durch die Vorschriften für den BND nıcht gedeckt. “ " th

Schließlich werden in den von Ex-Staatssekretär Reinhold Mercker im Auftrag der Großen Koalition erstellten Bericht über den BND diesem weiterhin vorgeworfents

- Fäischung von Nachrichten, die der Bundesregierung übermittelt wurden;

- "eklatante Mißachtung von Sicherhettsbestimmungenu; so war der später als Sowjet-Spion enttarnte Heinz Felfe ohne genaue Sicherheitsüberprüfung beim BND eingestellt worden. Felfes Zugehörigkeit zum Schwarzen Korps genügte zur Autnahme in die Agententruppe, wo zahlreiche SD- und Gestapoleute untergeschiüptt waren.";

- Vetternwirtschaft und Unterschlagungen: lukrative Jobs vergab Gehlen gern an eigene Angehörige und an Verwandte leitender Mitarbeiter. Dabei kam es sogar zu "Unterschlagungen in größerem Ausmaß" (Mercker: „ein korruptes Unternehmen«). Gehlen seibst versorgte beim BND allein 16 Verwandte.

- Nach einem „Vorwärtsu-Artikel, auf den sich der Extra-Dienst bezieht, scheinen selbst Geheimdienst-Morde nicht auszuschließen zu sein. ${ }^{46}$

Nicht zuletzt haben wır der Guillaume-Affäre die Einsicht zu verdanken, daß die bundesdeutschen Geheimdienste „nur relativ tüchtig sind - wie es R. Augstein dezent ausdrückt, ${ }^{47}$ oder, daß nach dem Urteil des CIA-Experten V. L. Marchetti, "der BND ein durch und durch unfähiger Geheimdienst $\alpha$ ist - +t8 möglicherweise weil BND wse VS ${ }^{\text {t9 }}$ soviel mit Aufgaben zu tun haben, mit denen sie ihrem Auftrag zufolge eigentlich nichts zu tun haben?

Alle Pannen und den Dilettantismus der bundesdeutschen Gehermdienste allein in der Guillaume-Affäre ım einzelnen zu beschreiben, würde Bände füllen. Deshalb soll hier nur eine kleine Auswahl aus den *Ermittlungen« gegen Guillaume genannt werden, um die oben zitierten Urteile überprüfbar zu machen:

- bei der unter Einschaltung des BFV und BND durchyeführten Sicherheitsüberprüfung Guillaumes fiel nicht auf, daỉ der Verlag "Volk und Wissen", bei dem Guillaume früher gearbeitet hatte, eine Tarnorganisation des DDRStaatssicherheitsdienstes war, obwohl sowohl dem BND so $^{3}$ als auch einer Abteilung des BfV ${ }^{1}$ entsprechende Erkenntnisse über den Verlag vorlagen. Die BfV-Abteilungserkenntnis befand sich jedoch nicht in der zur Uberprüfung herangezogenen Zentralkartei, die BND-Erkenntnis kam aufgrund mangelnder Kooperation zwaschen den Geheimdiensten nicht zum Tragen.

- Bei der Sicherheitsüberprüfung entging den Geheimdiensten weiterhin, daß Guillaume Im Mai 1966 schon enmal in eine Spionageaffäre verwickeit war, in die Sieberg-Affäre 52 .

- Ebenfalls war versäumt worden, eine im BFV bereits seit 1956 existierende Top-Quelle zur Uberprüfung Guillaumes heranzuziehen, die konkrete Verdachtsmomente enthielt und später zur Enttarnung beıtrug ${ }^{53}$.

it FR 11. 10.74.

4s Die folgenden Angaben sind zitiert nach dem - Stern $4+1 / 74$, S. is $8-160$. Zur Vetternwirtschaft vgl. auch DS $36 / 7+, 5.2+6$

to Extra-Dienst, 29. 10.74, S.

$\$ \mathrm{DS}+2 / 74$, S. 26.

t4 DS $+2 / 7+$, S. 169 .

ty Zu jen Praktiken des VS ryl. D. Damm, So arbeitet der Verfassungsschutz, Berlin 1970 ; ders.: Beruisverbot durch VS, in: Kritusche Justiz $3 / 73, \mathrm{~S} .+47-455$

su $\mathrm{FR}$ 31. 8. 74 .

31 FR 29. 8. 74

32 DS $19 / 74$, S. 23

53 Ebenda, S. 25 . 
- Bereits r958 meldete ein Doppel-Agent dem Hamburger Landesamt für VS (LFV), daß er Briefe des DDR-Staatssicherheitsdienstes für den damals in Frankfurt wohnenden G. Guillaume deponiere. Dem BFV war das nicht bekannt. Der früherc Doppel-Agent: "Die haben derart primitiv gearbeitet, die haben das sicher verschlafen und gar nicht weitergegeben. "54

- Als die Verfassungsschützer schließlich begannen, Guillaume zu überwachen, beschatteten sie zunächst tagelang die frühere Frankfurter Wohnung Guillaume's, weil sie nicht mitbekommen hatten, daß der Verdächtige längst in Bonn wohnte, wo cr übrigens auch im Telefonbuch stand. 55 - wie Weiland 1962, als in Düsseldorf der Spiegel-Geschäftsstellenleiter Erich Fischer als Rudolf Augstein verhaftet wurde, ${ }^{56}$ während Augstein wie gewöhnlich in Hamburg im Verlagshiaus war.

- Nach neun Monaten Uberwachung hatten die Verfassungsschützer schließlich herausgefunden, daß Guillaume zwei Söhne habe, was Willy Brandt gemeldet wurde, der zu Recht Ziveifel an den Verdächtigungen hatte, denn Guillaume hatte nur einen Sohn.

- Während Guillaume bereits überwacht wurde, konnte er dank der Unachtsamkeit der Geheimdienstler noch geheime Akten studieren und intime Kontakte mit der Sekretärin des Chefunterhändlers der Bundesregierung zur DDR, Gaus, anknüpfen. ${ }^{57}$

- Guillaumes Beschatter verhielten sich derart auffällig, daß Guillaume seine Beschattung erkannte und sich die Autonummern seiner Verfolger notrerte, woraufhin die Beobachtung für einige Wochen unterbrochen werden mußte. ${ }^{58}$

- Bei seinem letzten Frankreich-Urlaub sollen sich die Beschatter wiederum derart Jilettantisch verhalten haben, daß der Agent aufmerksam wurde und einen bislang noch nicht verhalteten Komplizen in der BRD habe warnen können. ${ }^{59}$

Nun muß man nach den bekannt gewordenen Praktiken der bundesdeutschen Geheimdienste froh sein, wenn sie nicht funktionieren - auch wenn für den mehr als 6000 Mitarbeiter umfassenden BND-Apparat ${ }^{60}$ und das ca. $\$ 3500$ bcamtete Staatsschützer und 13000 freiwillige V-Männers61 zählende BFV Hunderte von Millionen DM Jahr für Jahr verschleudert werden. So wie die Dinge liegen, muß man es auch begrüßen, daß BND, VS und Staatsschutzabteilungen der Polizei in der Vergangenheit eher gegeneinander gearbeitet als kooperiert haben ${ }^{62}$ und eher makaber mutet es an, wenn man verfolgt, wie die sozial-liberale Koalition in den letzten Jahren begonnen hat, den in Teilen völlig arbeitsunfähigen BND wieder funktionstüchtig zu machen, den VS durch enorme Geldspritzen aufzurüsten und die Kooperation zwischen den verschiedenen Geheimorganisationen zu effektivieren.

Wenn die BRD Weltmachtträumen abgeschworen hat, wozu bezahlt sie dann eigentlich BND-Agenten in Seoul, Saigon oder Hongkong, ${ }^{63}$ und wenn

$34 \mathrm{DS}_{40 / 74, S .}+4 ; \mathrm{FR}$ 1. 10.74

$53 \operatorname{DS}_{3} 8 / 74$, S. 28 .

36 $D S+2 / 7+$. S. 26

37 Ebenda, S. 28

SB FR 6. 12.74.

59 DS $19 / 74$, S. 21.

60 Stern v. 16.5 .74

"1 DS $22 / 74$. S. 24 .

t2 So der trühere Abreilungslester im BfY. Hermeniu, laut FR vom 29. 8. 74. Zu den ausgedehnten Machek ämplen zwischen BND und VS vil. DS $21 / 74$. S. : $5-29$.

63 Vgl. DS $21^{\prime} 7+$, S. 72 . 
bereits 1970 ein Anruf beim französischen Geheimdienst genügt hätte, Guillaume zu enttarnen, ${ }^{64}$ warum werden dann Mammutorganisationen deutscher Dunkelmänner ausgehalten, die eine gerwisse Effektivität eigentlich nur $\mathrm{m}$ Kampf gegen die politische Linke in der BRD entwickelt haben - bei der Vorbereitung von Berufsverboten, wie die AfV, ${ }^{65}$ bei der offenen Beschattung und das heißt Nötigung linker Oppositioneller, wie der Militärische Abschirmdienst, ${ }^{66}$ bei der politischen Denunziation, wie zuletzt spektakulär im Fall des FDPAbgeordneten Weber durch das Hamburger LfV, ${ }^{67}$ bei der Bespitzelung von Millionen Bürgern und dem Anlegen unkontrollierbarer Dossiers, wie alle Geheimdienste - im Zuge der Guillaume-Affäre bekannte der Berliner Innensenator Neubauer, daß allein das Berliner LFV Dossıers über insgesamt 190000 Personen führe...68. Natürlich weiß man, daß sie eben darum bezahlt werden, daß es eben das ist, was man hierzulande unter Schutz der freiheitlich-demokratischen Grundordnung versteht.

Allerdings hat die Guillaume-Affäre überdeutlich gezeigt, daß die Geheimdienste soyar von der Bundesregierung nicht zu kontrollieren sind, sondern ein ständiges außen- und innenpolitisches Risiko darstellen. Schliełlich ist über sie nicht nur ein Bundeskanzler gestürzt. So äußerte selbst ein Vertreter des Kanzlers über die Zusammenarbest zwischen BND und F. J. Strauß in der Guillaume-Aifäre: "Hier werden alte Rechnungen beglichen. Jetzt rächt sich der BND dafür, daß Ehmke damals die CSU-Zellen im Dienst knacken wollte. "69

So lehrt uns denn diese Guillaume-Affäre, dal sie die regierenden Frei- und Sozialdemokraten nichts lehren wird. Sie werden die Vetternwirtschaft im BND langsam abbauen - ca. I 30 funktionsiose BND-Verwandte wurden bereits gefeuert ${ }^{70}$ - und müglicherweise die Honorare für die Journalistenbestechung etwas kürzen. Sie müssen ja auch nicht bis zu is $000,-D M$ monatlich betragen. $\mathrm{Zu}$ keinem Zeitpunkt ist jedoch bisher der Wille deutlich geworden, die Dunkelmänner "abzuschalten* oder wenigstens drastisch zu reduzieren. Vielmehr schent der Trend dahin zu gehen, die Geheimdienste in technokratischen Sinne zu modernisieren und auszubauen, damit sie sich mit noch größerer Sorgfalt der Linken widmen können. Nach Konsequenzen gegen BND-Angehörige gefragt, die entgegen ihrem dienstlichen Auftrag in der BRD Politiker bespitzelt haben, antwortete die parlamentarische Staatssekretärin im Bundeskanzleramt, Marie Schlei, daß keine Disziplinarmaßnahmen eingeleitet werden sollen. Hier halten es die Sozialdemokraten wie die Unionschristen. Auf die Frage, was der seinerzeit für den BND zuständige CDU-Carstens auf die im MerckerBericht erwähnten strafrechtlichen Verfehlungen und "Unterschlagungen in erheblichem Ausmaß* hin unternommen habe, erklärte Carstens, er habe weinem Beamten damals seine Mißbilligung ausgesprochen - nichts weiter. $^{71}$

Zu fragen ist, welche Konsequenzen für einen demokratischen Staat es eigentlich hat, wenn unkontrolliert über Millionen Bürger geheime Dossiers aufgeüllt werden, wenn Geheimdienst-Dunkelmänner Berufsverbote vorbereiten, politi-

of DS $19 / 74$, S. 23 .

os Vyl. Kritische Justiz $3 / 73$, S. $4+7-455$

oo V'yl. Kritusche Justiz 2/74, vgl. auch DS $49 / 7+$, S. s8.

67 FR 31.8. und 6.9.74.

64 $F R+5.7+$.

o9 FR 6. 5. 74 .

70 DS $36: 7+$ S. $2+$

11 Fr 11. 10. 7+. Carstens weiter: Norinalerwetse trete er in solchen. Füllen für klare Konsequenzen ein, bes Gehermdienseen bestehe jedoch das Risiku, dals durch ölfentiche Gerichesverhandlungen Interna aus den Diensten bekannt würden. 
sche Organisationen von Spitzeln durchsetzt, Post und Telefon kontrolliert werden? Welcher Kontrolle unterliegt eigentlich ein Geheimdienst, in dem zwei Jahre nach Regierungsantritt der sozial-liberalen Koalition noch eine BNDAußenstelle Material gegen die SPD und vier Jahre danach ein BND-Agent Heysing noch Material über Presseverlage sammelt? Um eine Stellungnahme zu dieser Frage sollten sich Sozialisten in der SPD nicht herumdrücken.

Diethelm Damm 